\title{
Que vivent émotionnellement et pédagogiquement les externes en stage de médecine générale? Une étude qualitative
}

\section{What do pregraduate students in general practice experience in ambulatory clerkships? A qualitative study}

\author{
Céline BOUTON ${ }^{1}$, Isabelle RICHARD ${ }^{2}$, William BELLANGER ${ }^{1}$, Jean-François HUEZ ${ }^{1}$ \\ et François GARNIER ${ }^{1}$ \\ 1 Département de médecine générale, Faculté de médecine d'Angers, Université d'Angers, France \\ 2 Université d'Angers et Département de médecine physique et réadaptation adulte, Centre hospitalier régional \\ et universitaire d'Angers, France
}

Manuscrit reçu le 20 janvier 2012 ; commentaires éditoriaux formulés aux auteurs le 13 juin 2012 ; accepté pour publication le 21 décembre 2012

\begin{abstract}
Mots-clés
Externat ; médecine générale ; apprentissages cliniques ; ressenti

Résumé - Contexte et objectif : Le stage d'externat en soins primaires est devenu officiellement obligatoire dans les études de médecine depuis 1997 en France. La présence quasi permanente d'un enseignant clinicien auprès de l'étudiant et la mise en relation de l'étudiant avec des patients ambulatoires sont les spécificités majeures de ce stage. L'objectif de cette étude est d'appréhender le vécu, les perceptions et les apprentissages des étudiants dans le cadre de ce dispositif. Méthode : Une méthode qualitative recourant à des groupes de discussion focalisée avec analyse thématique a été utilisée, explorant les apprentissages et les impressions sur le stage et plus particulièrement la relation de l'étudiant avec l'enseignant et avec les patients. Résultats : Neuf étudiants ont participé à trois groupes de discussion. Les apprentissages cliniques ont été nombreux et variés. La relation médecin-patient a été une compétence fréquemment travaillée, grâce à l'observation du maître de stage par l'étudiant. Les étudiants ont découvert la médecine générale et sa place dans le système de soins. De nombreux sentiments les ont marqués : ennui, plaisir, lourdeur. Le dispositif pédagogique, initialement déstabilisant, a été perçu favorablement, grâce à l'observation directe de l'étudiant par le maître de stage et à la rétro-action qui est fournie. Les étudiants ont pu se projeter de façon argumentée dans leurs projets professionnels. Conclusion : Ce stage est propice à l' apprentissage de nombreux objectifs cliniques et à la découverte des soins primaires. L'observation directe de l'étudiant par le médecin et, à l'inverse celle du médecin par l'étudiant, sont des outils pédagogiques à exploiter au cours de ce stage.
\end{abstract}




$\begin{array}{ll}\text { Keywords } & \text { Abstract - Background and aim: Pregraduate ambulatory clerkships in medicine are now } \\ \text { Training; general } & \text { compulsory in France. Clerkships feature two major characteristics: the nearly constant pres- } \\ \text { practice; clerkship; } & \text { ence of clinical teachers to help students and student contact with ambulatory patients. The } \\ \text { clinical learning; } & \text { study was designed to determine students' experiences, perceptions and learning during the } \\ \text { feelings } & \text { training. Material and methods: A qualitative study based on focus groups was used and an } \\ & \text { interpretative analysis of the exchanges was carried out. The analysis examined learning and } \\ & \text { reactions related to training and more specifically the relation of students with teachers and pa- } \\ \text { tients. Results: Nine students took part in three focus groups. Clinical training was disconcert- } & \text { ing for students at first, but they appreciated it in the end. Students made significant clinical } \\ \text { learning progress. The improvement of the physician-patient relationship was mentioned fre- } \\ \text { quently thanks to the presence of teachers. Students realized how important the organization } \\ \text { of primary care and the place of general practitioners in the health care system were. They ex- } \\ \text { perienced a wide range of feelings, including boredom, enjoyment and cumbersomeness. Stu- } \\ \text { dents appreciated observing practitioners as well as being observed while benefiting from non- } \\ \text { judgmental feedback. They were able to better plan their professional projects. Conclusion: } \\ \text { This training helps the learning of clinical objectives and primary care. Direct observation of } \\ \text { students by teachers was the most important tool to be developed during clerkships. }\end{array}$

\section{Introduction}

En France depuis 1997, un stage de second cycle en médecine générale est devenu obligatoire et s'est progressivement mis en place dans les différentes facultés de médecine ${ }^{[1]}$. Plusieurs objectifs ont motivé la création de ces stages. Il s'agissait tout d'abord de promouvoir la filière de médecine générale aux yeux des étudiants, principalement pour être en mesure d'apporter à terme des solutions à des enjeux de démographie professionnelle. Les pouvoirs publics français, comme auparavant les américains, ont pris conscience de l'importance des stages d'externat dans les choix de carrière ${ }^{[2,3]}$. Un tel stage permet surtout la découverte de l'organisation des soins primaires et ambulatoires par les futurs généralistes et les futurs spécialistes. En plus des apprentissages dans le champ du « savoir-faire » (clinique, démarche décisionnelle...), les stages sont un temps privilégié de la formation pour que l'étudiant s'approprie des compétences relationnelles et celles liées au « savoirêtre », primordiales à sa professionnalisation ${ }^{[2]}$, plusieurs constats suggérant que certains apprentissages concernant la compétence relationnelle médecinpatient pourraient être plus faciles à effectuer dans une situation de consultation que dans les services hospitaliers.
Différentes organisations de ce stage de second cycle en médecine générale ont été proposées selon les facultés. Certains travaux ont évalué le vécu des externes dans ces stages à travers leurs attentes, leur satisfaction et leurs acquisitions cliniques ${ }^{[4,5]}$, sans forcément aborder explicitement ce qu'ils ressentaient et vivaient pendant le stage.

L'observation directe par un enseignant est un outil facilitant les apprentissages, qui fait souvent défaut dans le cursus de médecine des externes, ce qui est régulièrement rappelé dans la littérature ${ }^{[6,7]}$. Un tel dispositif pédagogique est facile à mettre en place en contexte de soins primaires, où peut être exploitée l'interaction d'un seul étudiant avec un seul enseignant durant le temps de la consultation. Le stage permet également la supervision et la rétro-action (feed-back) autour de situations cliniques souvent complexes. Le stage en médecine générale présente donc deux grandes particularités : l'une est le contact direct des étudiants avec des patients ambulatoires, dans leur contexte de vie ; l'autre est liée à la fréquence des situations où les étudiants sont placés dans la situation d'être observés en action par un enseignant. Ces deux particularités provoquent sans doute des apprentissages et des émotions spécifiques à ces situations et interviennent dans le processus de professionnalisation des étudiants. 
L'objectif de ce travail est d'explorer le vécu, les perceptions et les apprentissages des externes en stage de médecine générale en s'appuyant surtout sur deux spécificités de ce cadre de formation : le contact avec des patients ambulatoires dans leur position sociale habituelle, et l'interaction complexe et constante avec un médecin généraliste, comprenant des phases d'observation de l'un par l'autre et vice et versa.

\section{Matériel et méthode}

\section{Le dispositif de stage}

À la faculté de médecine de l'Université d'Angers, le stage de médecine générale est réalisé pendant une durée équivalente à celle des stages hospitaliers (neuf semaines), durant laquelle chaque étudiant accompagne un seul médecin généraliste. Ce stage accueille les étudiants de la troisième à la sixième année d'études, alors que les textes positionnent ce stage de la quatrième à la sixième année. Ce dispositif a été choisi pour des raisons de faisabilité et d'organisation. L'étudiant participe à toutes les activités du médecin (consultations, visites à domicile ou en institutions). Les objectifs institutionnels du stage sont l'apprentissage de compétences cliniques « transversales » : découverte des soins primaires ambulatoires, acquisition de compétences liées à la prise en charge globale du patient, relation médecin-patient, démarche clinique, et prévention en soins primaires. Ces objectifs sont adaptés au niveau de chaque étudiant. Un temps de supervision et de rétro-action est proposé pour chaque demi-journée de consultation. L'étudiant est fortement incité à réaliser seul un entretien ou un examen clinique par jour, qui sont suivis d'une reprise ultérieure en consultation à trois.

\section{L'étude}

Il s'agit d'une étude qualitative recourant à l'organisation de groupes de discussion focalisée (focus groups). La méthode qualitative a été choisie afin d'explorer l'ensemble des apprentissages et des émotions ressenties dans ce stage, y compris les plus inattendus ou les plus difficiles à mettre en évidence. Le groupe de discussion focalisée a pour objectif de croiser les points de vue et d'en élargir l'éventail grâce aux interactions entre les participants ${ }^{[8]}$.

Des groupes de discussion focalisée successifs ont été réalisés avec les mêmes étudiants en fin de stage de second cycle en médecine générale en juin 2010. Parmi les 25 étudiants qui effectuaient ce stage, à l'époque facultatif, neuf ont accepté d'y participer. L'intérêt de cette approche qualitative réduite à un seul groupe était de faciliter l'expression de leurs émotions et de leur vécu dans le détail. Les étudiants recrutés devaient être issus de promotions différentes et avoir des intérêts différents pour la discipline de médecine générale. Les rencontres ont eu lieu dans les locaux universitaires, en partie sur leur temps de stage.

Le guide d'entretien initial a été réalisé conjointement par le chercheur-animateur et le chercheur qui a ensuite analysé le verbatim, en s'appuyant sur la littérature et sur leurs hypothèses de recherche. Trois grands axes ont été explorés successivement dans les groupes de discussion focalisée : dans un premier temps les apprentissages et impressions sur le stage dans sa globalité, afin de dénouer les situations marquantes et de faciliter l'expression des étudiants ; puis leur regard sur leur relation avec leur maître de stage ; enfin les réactions produites par le contact avec des patients ambulatoires. L'animateur était formé aux entretiens semi-directifs et a posé des questions ouvertes préalablement préparées dans le guide (trois à quatre questions par groupe de discussion), en procédant à des relances appropriées en cas de besoin. Une analyse rapide entre chaque groupe de discussion permettait de réadapter le guide. Un observateur a enregistré l'ensemble de la discussion (enregistrement audio) et noté les expressions non-verbales. Le recueil de données a été arrêté lorsque la saturation des données a été atteinte sur l'analyse partielle du dernier verbatim réalisé.

L'enregistrement a été retranscrit mot à mot et le verbatim ainsi obtenu a été l'objet d'une analyse thématique de contenu par un seul chercheur de façon inductive, selon le principe de la théorie ancrée 


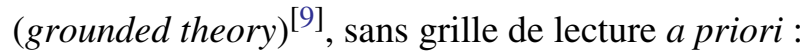
après plusieurs lectures globales, le texte a été découpé puis classé par thèmes et mot-clés. Les choix de catégorisation ont fait l'objet d'une discussion avec le deuxième chercheur sans qu'il n'intervienne ensuite dans la catégorisation elle-même. L'analyse a ensuite permis de faire ressortir de cette classification les apprentissages, émotions et notions exprimés. Les résultats ont été transmis aux interviewés quelques semaines après les entretiens pour qu'ils puissent signaler des erreurs ou d'autres idées oubliées.

\section{Les groupes de discussion focalisés}

Trois entretiens de groupes ont été menés, la saturation des données ayant été atteinte au troisème entretien. Le guide d'entretien prévu a été modifié entre le deuxième et le troisième groupe de discussion pour s'adapter aux données déjà recueillies. Les entretiens ont duré entre $1 \mathrm{~h} 12 \mathrm{~min}$ et $1 \mathrm{~h} 17 \mathrm{~min}$ chacun.

Neuf étudiants (sept femmes et deux hommes) ont participé aux trois groupes de discussion. Un étudiant était en première année du deuxième cycle des études médicales (D1), six en deuxième année (D2) et trois en troisième année (D3). Six déclaraient avant le stage être intéressés par la médecine générale, un n'était pas intéressé et deux ne savaient pas. Une bonne interaction entre les étudiants a pu être mise en place. Ils ont utilisé beaucoup d'exemples issus de leur stage. Tous les étudiants se sont exprimés même si quatre d'entre eux sont davantage intervenus, tandis qu'un étudiant est resté en retrait. Ils n'ont pas fait de remarques en relisant les résultats à distance.

\section{Résultats}

Les maîtres de stage

Dix maîtres de stage ont encadré les neuf étudiants pendant leur stage (un des étudiants avait deux maîtres de stage pour des raisons d'organisation de cabinet).
Ils avaient entre 33 et 61 ans, avec une moyenne d'âge de 43 ans (écart-type $=8,8$ ans, et médiane $=41$ ans) ; le sexe-ratio était de 1 . Tous exerçaient en zone urbaine ou péri-urbaine, dans un rayon de $16 \mathrm{~km}$. autour de la faculté de médecine.

Les thèmes abordés ont été regroupés en six grandes parties : le stage dans leur cursus, l'exercice de la médecine générale, les apprentissages cliniques, la relation médecin-étudiant, le patient, le ressenti des étudiants pendant le stage.

Le stage dans leur cursus

Les étudiants ont débattu relativement au meilleur moment pour réaliser ce stage, un stage précoce dans le cursus permettant de maitriser l'examen clinique «normal » plus tôt et « d'être à l' aise », mais risquant de déstabiliser l'étudiant par "manque de connaissances des pathologies ». Un des étudiants a souligné le fait que ce problème se retrouvait également dans les autres stages. E4 "C'est toujours pareil dans tous les stages,... tant que tu n'as pas fait la cardio, la pneumo, il y a beaucoup de stages où finalement tu ne comprends rien ... ».

E9 « ... si je l'avais fait en DI je l'aurais apprécié ce stage. Là en fin de D3 je l'apprécie vraiment (insistant) parce que c'est vrai qu'on a tout vu, on a une vue d'ensemble. Mais honnêtement [en fin de D3] je ne savais pas ce que c'était l'examen clinique normal d'un homme normal. ».

Régulièrement, les étudiants ont évoqué et analysé les différences qu'ils ont perçues entre la médecine hospitalière et la médecine générale : des pathologies, des stades et des prévalences différents en fonction du contexte de soins. E5 « à l'hôpital souvent c'est des choses plus graves, donc moins courantes».

\section{L'exercice de la médecine générale}

Les conditions d'exercice de la médecine générale ont été largement décrites depuis l'aspect financier, l'organisation de la vie personnelle, la formation 
médicale continue, jusqu'aux champs de compétences. Les étudiants ont pris en compte la possibilité de se donner du temps, en revoyant certains problèmes à distance. E2 «ça apprend à cadrer une consultation... «on va s'arrêter là, vous reviendrez la semaine prochaine on continuera. », ça à l'hôpital finalement nous on le fait jamais. On a trois patients à voir dans la matinée...».

Un débat s'est instauré car certains ont pu trouver des « redondances » et de l'ennui dans l'activité du médecin généraliste, tandis que d'autres ont trouvé l'exercice "particulièrement varié ». E4 « une phase où ça me gonflait un peu, de voir... toujours un peu la même chose ». E3 « je ne suis pas tout à fait d'accord, parce que je trouve que la médecine générale c'est très très varié ». E4 "c'est toujours la visite des 1 mois du nourrisson, des 2 mois, des 3 mois, le mal de dos... ». E3 « Oui mais tu passes [...] de la gynéco à la pédiatrie, à la rhumato. ».

Pour certains étudiants l'exercice de la médecine générale est apparu solitaire, d'autres ont au contraire perçu les possibilités de collaboration avec d'autres professionnels de santé. Ils ont pu décrire dans leurs exemples les différents rôles du généraliste et sa place centrale dans le système de soins. E9 "je ne me vois pas tout seul dans un cabinet E3 «Non aujourd'hui on parle de cabinets où il y a plusieurs médecins, avec une équipe paramédicale, éventuellement... ». E9 "Oui mais honnêtement combien de fois dans ta journée le médecin généraliste quand il ne savait pas, il est allé voir ses collègues?».

\section{Les apprentissages cliniques}

Les étudiants ont décrit de nombreuses situations cliniques issues de différents champs disciplinaires. Ils ont pu percevoir les notions de prévalence des pathologies en médecine générale. Ils ont profité de ce stage pour « approfondir l'examen clinique », notamment en examinant «l'homme normal », ainsi qu'en réalisant des « examens ciblés». Certains étudiants ont pu travailler la démarche diagnostique et décisionnelle et observer la gestion de situations d'incertitude qui se présentent régulièrement lors de la prise en charge de problèmes indifférenciés. E6 «dans les serviceson a plus ou moins une idée du diagnostic, donc on fait un examen clinique complet, parce qu'on nous le demande, mais on ne va pas chercher plus loin, on ne va pas chercher d'autres diagnostics différentiels, ... mais en médecine générale, il faut trouver ce qu'il a, et cibler les questions par rapport aux symptômes ».

Ils ont été familiarisés avec la prise en charge de pathologies chroniques et avec le suivi au long cours. Une étudiante a pu exprimer l'importance de réaliser des apprentissages contextualisés.

\section{La relation médecin-étudiant}

Au sujet du dispositif de stage les étudiants ont été en majorité enthousiasmés par l'accueil qui leur a été fait et parlent de «bon contact», "d'échanges», de "disponibilité » malgré une première période d'adaptation pendant laquelle ils se sentaient moins à l'aise. Ils ont des avis divergents sur leur autonomie pendant le stage. Certains estiment faire plus de choses que dans d'autres stages, d'autres trouvent qu'ils perdent en autonomie et restent en retrait. Ces perceptions varient selon eux en "fonction du médecin», de "la place qu'il leur donne dans la consultation », de sa «confiance » en eux.

Le fait d'être «observé et jugé » par l'enseignant a été évoqué mais n'a pas été vécu de façon désagréable par les externes car les maitres de stage les ont mis dans la plupart des cas à l'aise. Certains ont été vexés d'être «critiqués » ou «jugés »dans un premier temps puis y ont trouvé leur intérêt, notamment dans le retour sur leur pratique clinique : "être observé et repris sur un examen clinique ». À l'inverse les étudiants se sont retrouvés dans la situation d'observer le médecin et de critiquer sa pratique. Cette critique du maître de stage a même été dans certains cas motivée par le médecin lui-même, accordant à l'étudiant une réflexion et une place privilégiée. $E 4$ «Moi je trouve qu'au début on se sent jugé, mais en fait si on fait quelque chose de "pas bien", on va nous reprendre mais gentiment, ... c'est vraiment pour nous aider, pour nous faire progresser ». E1 «On a pas vraiment le sentiment d'être jugé quoi, c'est plus observé le terme à utiliser que jugé ». 
Le patient

Un étudiant a utilisé l'adjectif « sauvage » pour parler des patients ambulatoires qui, n'étant pas captifs d'une institution, auraient des "idées bien arrêtées » et seraient «moins dociles » aux propositions médicales, ce qui expliquerait en partie les problèmes d'observance parfois rencontrés. Les étudiants n'ont pas exprimé de difficultés particulières à être en face d'un patient ambulatoire dans son contexte de vie habituel.

La principale difficulté du stage a été l'interaction à trois en consultation, ainsi que le positionnement de l'étudiant au milieu d'une relation, souvent ancienne, entre le médecin et son patient. Les étudiants ont ressenti «une gêne » dans les sujets intimes, dans les situations où le médecin les corrigeait devant le patient. Ils se sentent parfois peu crédibles aux yeux du patient. L'échange à trois complique ainsi la consultation mais ils énoncent des stratégies permettant d'y remédier : l'enseignant rappelle le rôle de l'externe et adapte son cabinet, l'étudiant se met « en retrait », la discussion s'engage « après le départ $d u$ patient », ils utilisent «l'ordinateur » pour communiquer par des notes, une éventuelle séparation des pièces du cabinet... L'étudiant peut avoir un rôle actif dans la consultation en demandant des éclaircissements sur un point compliqué pour lui et pour le patient ou en rappelant un oubli. E1 «ça m'est arrivé plusieurs fois de poser des questions à la personne et finalement elle regarde le médecin et elle lui répond à lui. ». E4 « je trouve ça plus facile quand on est seul face à la personne parce qu'on n'a pas l'impression d'être jugé sur ce qu'on va dire ».

La relation entre le médecin et son patient a pris une part importante de la discussion lors des entretiens. C'est, selon les étudiants, le principal apprentissage qu'ils aient fait. Ils ont largement décrit cette relation au long cours, basée sur la confiance, l'écoute et la communication, et tenant compte de la globalité et de l'intimité du patient. Ils ont rappelé que cette relation s'appliquait également à tous les médecins qui suivent des patients chroniques en ambulatoire. Poser des questions intimes et aborder des sujets « délicats » leur semble difficile. Ils ont pu observer les médecins généralistes poser des questions intimes avec naturel et sans peur, ce qui permet au patient de s'exprimer. Ils ont pu s'exercer à "l'éducation $d u$ patient » et voir leur maitre de stage négocier avec celui-ci. Enfin pour certains la communication avec le patient est passée par un certain degré de «papotage », d'allure anodine mais parfois sémiologiquement très enrichissant. Ils ont pointé de façon marquée la nécessité pour le médecin de "se protéger » et de garder une distance adaptée dans la relation au patient. Par ailleurs, ils ont été mis en situation de communiquer eux-mêmes avec le patient. Ils ont pu avoir un retour sur leurs paroles, leurs comportements et leurs attitudes grâce à l'observation directe par leur maître de stage. E9 "C'est le seul stage où on a eu un médecin tout le temps avec nous, et qui nous dit le positionnement qu'on doit avoir par rapport au patient. Comment se comporter, notre approche, parce qu'on fait beaucoup d'examens cliniques à l'hôpital, beaucoup d'interrogatoires, mais on prend peu conscience de nos paroles, comme personne ne nous reprend ou nous explique comment aborder les choses. Pour moi c'était le plus important».

\section{Ressenti des étudiants pendant le stage}

Les étudiants se sont initialement sentis «déboussolés » du fait du changement de cadre du stage. Ils ont vécu de façon difficile les consultations «psychologiques » où l'écoute d'un patient et de ses soucis peut amener à une certaine pesanteur. Ils ont exprimé la difficulté d'entendre des choses lourdes, douloureuses, «pas rigolotes » et de «s'identifier » plus ou moins au patient. E2 «il y avait beaucoup de choses que je gardais, et qui étaient lourdes et j'avais une impression vraiment de plombage. ». À l'inverse ils ont pu se sentir à l'aise et détendus quand ils ont jugé que la situation le permettait : examen du nourrisson, certificat de sport, patient qui plaisante...

Globalement ils ont exprimé un fort intérêt pour ce stage, même si parfois certains ont vécu des phases «d'ennui » notamment à mi-parcours. L'ennui a été exprimé surtout par les plus jeunes qui ont eu moins de responsabilités et moins d'implication dans la démarche décisionnelle. Ils ont, dans différentes situations, ressenti de la «frustration ». Tout d'abord 
celle de faire de la "bobologie », de faire un peu de tout mais «sans aller au bout des choses ». Enfin, l'attitude de certains patients refusant de suivre les prescriptions malgré leur insistance a pu les déstabiliser. Ils ont compris que poser une question n'est pas un problème mais qu'il faut ensuite "gérer la réponse » du patient, qui parfois peut déborder ou surprendre l'étudiant et nécessite de sa part une compétence et des connaissances qu'il n'a pas encore. Certains n'ont pas pu prendre beaucoup de libertés mais la majorité a pu poser des questions aux patients, «proposer des traitements ou des bilans », faire des remarques au médecin.

Leur discours a montré plusieurs fois une ambiguité entre leur position d'étudiant et la position de médecin dans laquelle ils se projettent. Il leur arrive de faire des lapsus ou des raccourcis en se nommant médecin par anticipation. E1 «j'étais parti dans la consult et j'ai proposéde faire un petit bilan et tout. ». Ce stage a permis aux étudiants d'avancer dans leur réflexion professionnelle et d'alimenter positivement ou négativement leur attrait pour la discipline. E9 "Moi j'ai ressenti que c'était vraiment pas fait pour moi, ... à l'origine j'avais un a priori en me disant ça m'attire pas. Je fais ce stage pour voir si au final je me trompe, pour savoir pourquoi ça ne m'attire pas. Et au final j'ai compris, il faudrait que j'invente moimême une pratique totalement différente de celle que j'ai vue, et je ne sais pas si c'est réalisable.».

\section{Discussion}

Les résultats de ce travail englobent l'ensemble des objectifs institutionnels du stage, le premier objectif étant les apprentissages cliniques, ainsi que la découverte du contexte des soins primaires ambulatoires. Comme dans le travail de Renoux ${ }^{[5]}$, les apprentissages cliniques ont été nombreux variés, contextualisés, même s'ils ne sont pas spécifiques de ce stage mais favorisés par le contexte de la consultation en soins primaires. Les étudiants ont particulièrement insisté sur leur découverte de la relation médecinpatient, qui n'est pas l'apanage du seul médecin généraliste, mais celui-ci est dans une position privilégiée de suivi au long cours des patients. Ils ont bien analysé le changement de cadre potentiellement déstabilisant proposé par le stage.

L'analyse inductive du verbatim a permis de répondre à l'objectif principal et original de ce travail en décrivant les émotions ressenties par les étudiants. Les sentiments exprimés étaient à la fois contradictoires et marqués. Chaque étudiant a vécu différemment son stage en fonction de son parcours précédent. L'amplitude des sentiments a été importante chez les mêmes étudiants : ils ont pu passer dans un même stage de la frustration au plaisir, de l'ennui à l'intérêt, de la lourdeur à la légèreté. La proximité du patient et du médecin a pu probablement exacerber ces sentiments. La gêne a été ressentie pour les situations intimes. Devant la lourdeur de certaines situations, ils ont bien souligné l'importance pour le médecin de se protéger.

La limite principale de ce travail est liée à un déficit de triangulation, qui ne permet pas de garantir une crédibilité optimale des résultats. Ainsi, concernant la triangulation des chercheurs, même si le deuxième investigateur-animateur a participé à l'analyse, il a discuté uniquement les choix de catégorisation et l'analyse n'a été réalisée que par un seul investigateur; il n'y a par ailleurs eu ni triangulation des sources d'information (seuls des étudiants ont été interrogés) ni triangulation des méthodes (l'étude a exploité exclusivement des groupes de discussion focalisée, alors qu'elle aurait pu être complétée par des entretiens individuels ou par le recours à une observation participante). En revanche, il y a eu une validation par les répondants et la saturation des données a été atteinte, aucune idée nouvelle ne ressortant à la fin du troisième entretien, ces deux éléments concourant à la crédiblité des résultats.

Il faut également admettre certaines limites concernant la transférabilité des résultats, les caractéristiques spécifiques de la faculté, des neufétudiants et de leurs maîtres de stage, ainsi que le volontariat pour participer aux groupes de discussion ne permettant pas de généraliser sans réserve les résultats.

Les maîtres de stage de cette étude ne correspondent pas aux caractéristiques sociodémographiques habituelles des médecins généralistes : ils sont plus jeunes et ce sont plus fréquemment des femmes. Ceci 
s'explique par une volonté de la faculté d'avoir un pool de maitres de stage jeunes pour les stages de second cycle. De plus, ils exercent plutôt en secteur urbain pour faciliter l'accès aux étudiants mais l'augmentation des besoins en lieu de stage éloigne progressivement ceux-ci de la faculté.

L'évaluation de leur satisfaction n'étant pas l'objectif de ce travail, le volontariat n'a probablement que peu de répercussion sur les résultats, hormis un engouement sans doute surexprimé. Le fait que les organisateurs (animateur et observateur) soient les responsables du stage aurait pu limiter l'expression des avis non consensuels et « institutionnaliser » ces entrevues, ce qui ne se ressent pas dans l'analyse du verbatim.

Sur un plan pédagogique, ce stage permet une contextualisation des connaissances et des capacités des étudiants dans des situations cliniques authentiques. De plus, de par le ratio un étudiant pour un médecin, ils sont mis en situation d'être observés et de recevoir une rétro-action mais aussi d'observer le maître de stage, qui peut alors devenir un modèle de rôle ${ }^{[10]}$. Les étudiants ont apprécié d'être observés sans se sentir jugés et ce, notamment, dans le champ de la relation médecin-patient. Cet apprentissage semble facilité par la contextualisation et le dispositif de la consultation; en effet la relation médecinpatient est un domaine de formation difficilement accessible en dehors des situations réelles de soins. L'observation d'un médecin en train de communiquer avec son patient permet d'élaborer une modélisation de cette relation. L'expérimentation par l'étudiant de la communication avec le patient et la rétro-action qu'il reçoit sont vécus positivement par les étudiants.

La littérature s'accorde sur l'importance du modèle de rôle et de l'observation directe des étudiants par les enseignants, ainsi que sur la rareté de ces situations d'apprentissage dans le système de formation hospitalo-universitaire français ${ }^{[6,7,11]}$. Ces situations pédagogiques sont fondamentales dans un objectif de professionnalisation et de certification des compétences ${ }^{[10,12]}$. La consultation semble donc être un lieu privilégié pour créer ces situations pédagogiques et semble transposable dans un contexte de soin spécialisé ambulatoire, hospitalier ou libéral. Ce constat peut être une solution à la surcharge des services hospitaliers, liée à l'augmentation actuelle du numerus clausus, en soutenant l'ouverture de nouveaux lieux de stage ambulatoires. La relation de confiance entre le maître de stage et l'étudiant semble avoir favorisé l'échange et la réflexion autour de l'action réalisée, expliquant en partie la satisfaction des étudiants.

Le débat qui s'est instauré quant au meilleur moment dans le cursus pour faire ce stage pourrait être développé à propos de nombreux stages et les enseignants se posent régulièrement cette question. En début de cursus, les étudiants manquent de connaissances pour élaborer des hypothèses et une démarche décisionnelle. En fin de cursus, ils ont envie d'être plus autonomes. Les étudiants sont arrivés eux-mêmes à la conclusion qu'il n'y avait pas forcément de moment plus approprié pour faire ce stage. Cette conclusion conforte l'idée que ce stage peut être offert à différents moments du cursus mais que cela nécessite une adaptation des objectifs au niveau de l'étudiant.

La proximité avec les médecins a permis aux externes de réfléchir à ce que représentait l'exercice médical sur un plan professionnel, social et personnel. La modélisation créée permet aux étudiants de se projeter dans leurs futurs choix de carrière en toute connaissance de cause. Même si cette découverte semble indispensable, l'intérêt du stage n'est pas de motiver tous les étudiants à la médecine générale mais bien d'appréhender le dispositif des soins primaires, dans lequel ils exerceront ou avec lequel ils collaboreront forcément au sein du système de santé. La comparaison permanente dans le verbatim entre les mondes hospitalier et ambulatoire montre comment les étudiants ont repéré le rôle de chaque professionnel de santé. Les étudiants dans ce stage semblent se sentir davantage médecins et leurs hésitations sur leur place d'étudiant ou de soignant ainsi que les libertés qu'ils ont prises le suggèrent. La littérature a déjà signalé que les patients sont en général assez satisfaits d'être vus par ou en présence d'un étudiant et cela ne parait pas être un obstacle à la consultation ${ }^{[13]}$.

Les maitres de stage ambulatoires de la faculté ont une obligation de formation annuelle en pédagogie. Certains des éléments discutés dans cette étude doivent sans doute être au cœur de ces formations. Tout 
d'abord, il semblerait important de mieux prendre en compte les sentiments, parfois violents et difficiles, ressentis par les étudiants devant des situations délicates, pour ne pas mettre certains étudiants en difficulté. De plus, un travail sur les outils pédagogiques semble indispensable. La perception par les maîtres de stage de ce en quoi ils sont des modèles de rôle n'est sans doute pas toujours évidente, bien qu'elle soit nécessaire à l'efficacité de cet outil pédagogique fort ${ }^{[14]}$. De même, si l'observation directe de l'étudiant par le médecin semble inhérente au dispositif de stage, la rétro-action qui l'accompagne doit également faire partie des appropriations des maîtres de stage. L'acquisition de ces outils pédagogiques par les médecins est indispensable pour que les étudiants ne soient pas dans une situation de compagnonnage simple et d'apprentissage par «imbibition», mais bien dans un « compagnonnage cognitif » ${ }^{[15]}$. Enfin nous avons vu que la consultation à trois, « médecin, patient et étudiant», déstabilise sans doute les trois protagonistes. Des stratégies d'adaptation ont été décrites par les externes et mériteraient d'être travaillées et mutualisées lors des formations des maîtres de stage. Un travail qualitatif explorant ce que les médecins vivent quand ils accueillent un étudiant permettrait de croiser les points de vue.

Le stage est devenu obligatoire dans notre faculté pour les étudiants entrés en première année du deuxième cycle des études médicales en 2010 et il paraitrait intéressant de refaire un tel travail avec des promotions d'étudiants «non-volontaires ». Le vécu et le ressenti des externes durant leurs autres stages est également un domaine inexploré en recherche qualitative, qui pourrait faire un parallèle enrichissant avec ce travail.

\section{Conclusion}

Pendant le stage d'externat en médecine générale, les étudiants semblent avoir été mis en situation de travailler l'ensemble des objectifs institutionnels. Ils ont pu fréquemment percevoir de façon concrète la notion de « relation médecin-patient ». Ils ont bien analysé le changement de cadre, potentiellement déstabilisant, proposé par le stage et ont exprimé des sentiments contrastés. Ils ont été mis en situation d'être observés et de recevoir une rétro-action sans se sentir juger. Enfin leur discours a permis de mettre en évidence le modèle de rôle du maître de stage, qui est observé par l'étudiant. Les outils pédagogiques du stage mis en évidence doivent faire l'objet de formations spécifiques approfondies pour les maîtres de stage.

\section{Contributions}

Isabelle Richard, François Garnier et William Bellanger organisent le stage ambulatoire en second cycle et ont apporté leurs réflexions à ce travail. François Garnier et Céline Bouton ont réalisé le recueil et l'analyse des données. Jean-François Huez a procédé à une révision et une correction attentive du manuscit. Les auteurs remercient particulièrement les étudiants qui ont participé et les enseignants cliniciens qui les encadrent.

\section{Approbation éthique}

Le protocole d'étude a été examiné par le conseil d'administration du département de médecine générale de la Faculté de médecine et ce dernier n'a pas soulevé d'objection éthique.

\section{Déclaration d'intérêts}

Les auteurs déclarent qu'ils n'ont pas de conflit d'intérêt financier en lien avec l'objet de cet article. Ils déclarent tous une position hiérarchique à l'égard des étudiants et des médecins maîtres de stage, dans le cadre de leurs responsabilités institutionnelles au sein de la faculté, relativement à l'organisation du stage ou dans l'enseignement.

\section{Références}

1. Le Neel H, Honnorat C, Grosbois B, Chapron A. Un stage en médecine générale au cours du $2^{\mathrm{e}}$ cycle. Expérimentation à la faculté de médecine de Rennes. 
La Revue du praticien - Médecine générale 2007;21:407-9.

2. Barrier JH, Brazeau-Lamontagne L, Colin R, Quinton A, Llorca G, Ehua FS et le conseil pédagogique de la Conférence Internationale des Doyens des Facultés de Médecine d'Expression Française. La formation au professionnalisme des futurs médecins. Recommandations du Conseil Pédagogique de la CIDMEF. Pédagogie Médicale 2004;5:75-81.

3. Lefèvre JH, Karila L, Kernéis S, Fiessinger J-N, Rouprêt M. Désintérêt des futurs médecins pour la médecine générale. Résultats d'une enquête nationale sur les choix de spécialisation auprès de 1870 externes français. Presse Med 2010;39:e58-e65.

4. Renoux C, Lehr Drylewicz AM, Robert J, Potier A, Huas D, Lebeau JP. Les attentes des externes sur le stage en médecine générale en deuxième cycle à la faculté de médecine de Tours. Exercer 2009(87):80-4.

5. Renoux C, Lehr-Drylewicz AM, Huas D, Lebeau JP. Évaluation préliminaire d'un stage d'externat expérimental en médecine générale à la faculté de médecine de Tours. Pédagogie Médicale 2009;10:175-83.

6. Langevin S, Hivon R. En quoi l'externat ne s'acquitte$\mathrm{t}$-il pas adéquatement de son mandat pédagogique? Une étude qualitative fondée sur une analyse systématique de la littérature. Pédagogie Médicale 2007;8:7-23.

7. Russell G, Ng A. Taking time to watch: Observation and learning in family practice. Can Fam Physician 2009;55:948-50.
8. Gibbs, Anita. Focus groups. Social research update 19: University of Surrey. 1997. [On-line] Disponible sur: http://sru.soc.surrey.ac.uk/SRU19.html.

9. Guillemette F. L'approche de la grounded theory pour innover? Recherche qualitative 2006;26:32-50.

10. Chamberland M, Hivon R. Les compétences de l'enseignant clinicien et le modèle de rôle en formation clinique. Pédagogie Médicale 2005;6:98-111.

11. Jouquan J. Pour ne pas en rester aux déclarations d'intention concernant le compagnonnage pendant l'externat. Pédagogie Médicale 2007;8:5-6.

12. O’Brien HV, Marks MB, Charlin B. Le feedback (ou rétro-action) : un élément essentiel de l'intervention pédagogique en milieu clinique. Pédagogie Médicale 2003;4:184-91.

13. O’Malley PG, Omori DM, Landry FJ, Jackson J, Kroenke K. A prospective study to assess the effect of ambulatory teaching on patient satisfaction. Acad Med 1997;72:1015-7.

14. Côté L, Leclère H. How clinical teachers perceive the doctor-patient relationship and themselves as role models. Acad Med 2000;75:1117-24.

15. Jouquan J, Bail P. À quoi s'engage-t-on en basculant du paradigme d'enseignement vers le paradigme d'apprentissage ? Pédagogie Médicale. 2003;4:163-75.

Correspondance et offprints: Céline Bouton, Département de médecine générale, Faculté de médecine d'Angers, 49045 Angers Cedex, France.

Mailto : celine.bouton@univ-angers.fr 